spectively). $63 \%$ of respiratory patients never spoke about their sexuality with a health professional. $60 \%$ would like the health professional to begin to talk about their sexuality. $36 \%$ of patients spoke about it with a professional. In this group, $94 \%$ of patients told the professional about their sexual activity on their own initiative.

Conclusion: More than one of two chronic respiratory disease patients $(77 \%)$ participating in a rehabilitation program want sexuality to be taken into consideration during their program.

\section{Fatigability of Lower Limb Muscles during Walk- ing in Chronic Obstructive Pulmonary Disease}

Marquis Nicole, Centre de recherche, Hôpital Laval. Québec

Bouyer Laurent, Centre interdisciplinaire de recherche en réadaptation et intégration sociale (CIRRIS), Québec

Debigaré Richard, Laviolette Louis, Brouillard Cynthia, Centre de recherche, Hôpital Laval. Québec

Saey Didier, Université Catholique de Leuven, Leuven, Belgique

Maltais François, Centre de recherche, Hôpital Laval. Québec

Background: Patients with chronic obstructive pulmonary disease (COPD) perceive much less quadriceps fatigue during walking compare to cycling. Whether other lower limb muscles could develop fatigue during walking is unknown. The purpose of this study was to assess the electrical activity of five lower limb muscles during a 6-minute walking test in 11 healthy subjects and in 10 patients with COPD matched for age and activity level.

Methods: Surface electromyographic (EMG) data were recorded in five muscle groups (soleus, gastrocnemius (GM), tibialis anterior, vastus lateralis and rectus femoris) of the right leg during the walking test. The EMG median frequency of all contractions at minute 2 and 6 were averaged for each muscle group. Ventilation, oxygen consumption and $\mathrm{CO} 2$ production were also continuously measured throughout the test.
Results: Although the walking distance $(494 \pm 116$ vs. $625 \pm 50 \mathrm{~m} ; P<0.01)$ and the walking speed (1.7 \pm 0.4 vs. $\left.2.1 \pm 1.2 \mathrm{~m} \cdot \mathrm{s}^{-1} ; P<0.01\right)$ were reduced in COPD compared with controls, patients worked at a higher percentage of their estimated maximum voluntary ventilation during the test $(118 \pm 32 \%$ vs. $51 \pm$ $14 \% ; P<0.01)$. The time course of the EMG median frequency from minute 2 to 6 differed between patients with COPD and healthy controls for the soleus, GM and tibialis anterior suggesting the occurrence of a muscle fatiguing profile in COPD.

Conclusions: Evidences of a fatiguing profile was found in three lower limb muscle groups during walking in COPD despite a slower walking speed compared to healthy controls.

\section{Pulmonary Rehabilitation Results in Significant Effect on Physical Outcomes: Preliminary Data from the Saint John Regional Hospital, New Brunswick, Canada}

Mundle J. Stephen, Saint JohnRegionalHospital, Saint John, New Brunswick, Canada

Christopher M. Blanchard, DalhousieUniversity, Halifax, Nova Scotia, Canada

Background: Current guidelines claim that pulmonary rehabilitation is a cost effective method for increasing exercise tolerance, decreasing hospitalization, and improving knowledge and quality of life in patients with chronic obstructive pulmonary disease. The purpose of the present study was to examine the changes in physical and psychological outcomes during and after completion of a pulmonary program.

Methods: Between September 2004 and April 2006, 27 patients attended pulmonary rehabilitation. All patients were referred by a Respirologist and had moderate to severe respiratory impairment on pulmonary function testing. They attended 3 supervised exercise sessions per week for 10 weeks under the supervision of a nurse and physiotherapist. Education modules were provided by the physiotherapist and respiratory therapist to increase knowledge and independence with disease 